\title{
Opfer zweiter Klasse: Opfer staatlicher Gewalt und die Beschwerde in Strafsachen
}

Moritz Oehen *

Privatkläger sind zur Beschwerde in Strafsachen legitimiert, sofern sie zivilrechtliche Forderungen geltend machen. Personen, die Forderungen gegen das Gemeinwesen geltend machen, sind hingegen nicht legitimiert. Das Bundesgericht hält an dieser Rechtsprechung aus verschiedenen Gründen im Grundsatz fest.

I. Einleitung 35

II. Die Legitimation der Opfer staatlicher Gewalt vor Bundesgericht .....................35

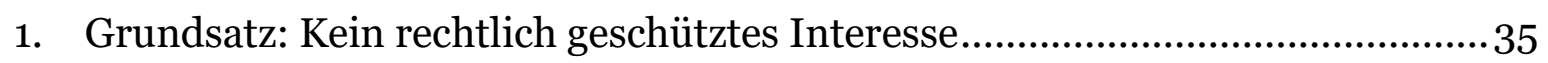

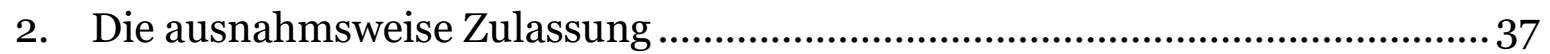

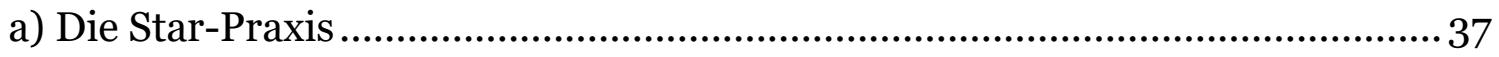

b) Die erweiterte Star-Praxis: Der Fall Assenov..............................................38

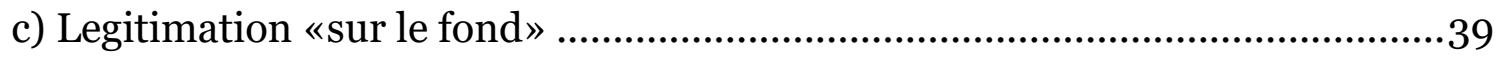

III. Konsequenzen für das Opfer staatlicher Gewalt ..............................................40

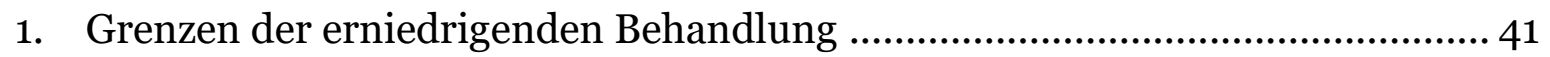

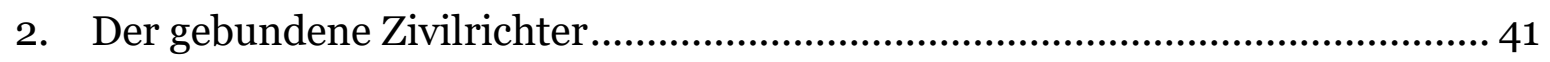

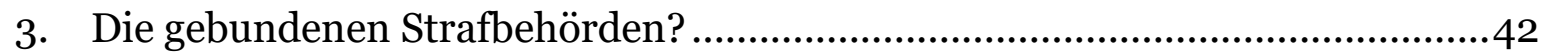

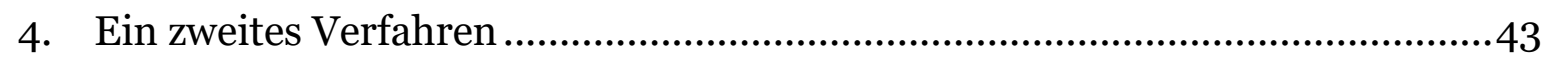

IV. Gerechtfertigte Ungleichbehandlung? ......................................................44

1. Das Strafmonopol des Staates...............................................................44

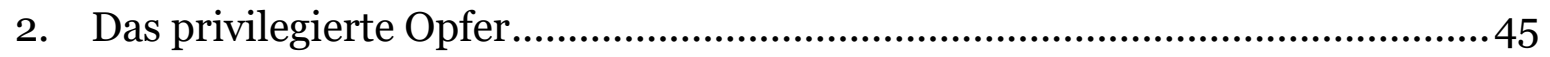

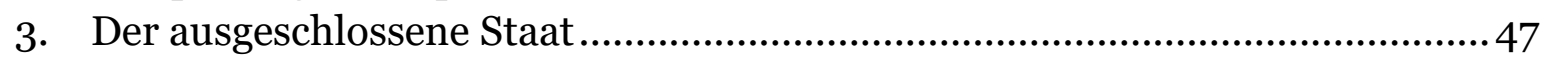

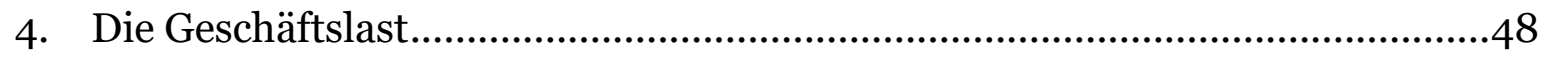

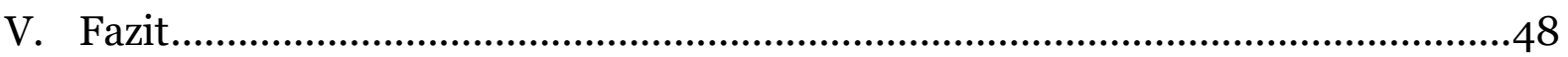

Zitiervorschlag: Moritz Oehen, Opfer zweiter Klasse: Opfer staatlicher Gewalt und die Beschwerde in Strafsachen, in: sui-generis 2015, S. 34

URL: $\quad$ sui-generis.ch/12

DOI: $\quad$ https://doi.org/10.21257/sg.12

* Moritz Oehen, MLaw, wissenschaftlicher Assistent, Universität Zürich. 


\section{Einleitung}

1 Zur Illustration der Thematik dieses Aufsatzes sei ein kurzes Beispiel angeführt: In einem Spitalzimmer liegen zwei Verkehrsunfallopfer, mit identischen Verletzungen; zwei Fussgänger, der eine von einem privaten PW angefahren, der andere von einem Streifenwagen der Polizei. Die beiden Fussgänger befinden sich tatsächlich in der gleichen Situation - rechtlich hingegen ist das Opfer, das vom Streifenwagen angefahren wurde, zumindest vor Bundesgericht ein Opfer zweiter Klasse. Ein absurdes Beispiel? Ja, womöglich. Eine verwegene Feststellung? Mitnichten!

Seit der Volksinitiative «zur Entschädigung der Opfer von Gewaltverbrechen» ${ }^{1}$ und der darauf gründenden ersten Version des $\mathrm{OHG}^{2}$ wurde die strafprozessuale Rolle des Opfers aufgewertet: War das Opfer im reformierten Strafprozess des vergangenen Jahrhunderts in erster Linie Tatzeuge im Sinne eines Beweismittels, 3 räumte ihm spätestens die gesamtschweizerische Strafprozessordnung spezifische Schutzrechte und - unter dem Begriff der «Privatklägerschaft» - Parteirechte ein (Art. 107 i.V.m. Art. 104 StPO). Das rein dienende Opfer wurde zum mitbestimmenden Verfahrenssubjekt, das auf Augenhöhe mit Beschuldigtem und Staatsanwalt steht. Diese Bestrebungen zugunsten des Opfers können allerdings nicht darüber hinwegtäuschen, dass noch immer punktueller Verbesserungsbedarf existiert.

\footnotetext{
BBl 1980 III 1287.

2 Bundesgesetz vom 4.10.1991 über die Hilfe von Opfer von Straftaten (Opferhilfegesetz).

3 Eingehend zur Stellung im reformierten Strafprozess: Daniela Verena Jabornigg, Die Stellung des Verletzten in den schweizerischen Strafprozessordnungen zwischen Beweismittel und Partei, Diss. Basel 1998, Basel 2001, 284 f.
}

3 Um die einleitende Feststellung nachvollziehbar zu machen, gehe ich zunächst auf die Legitimation von Opfern staatlicher Gewalt vor Bundesgericht ein (II.), bevor ich die Konsequenzen der höchstrichterlichen Rechtsprechung aufzeige (III.). Die Argumente des Bundesgerichts sollen schliesslich näher betrachtet und bewertet werden (IV.).

\section{Die Legitimation der Opfer staatli- cher Gewalt vor Bundesgericht}

4 Die Legitimation zur Beschwerde in Strafsachen ans Bundesgericht knüpft an zwei Voraussetzungen an: Erstens ist die Teilnahme am vorinstanzlichen Verfahren verlangt (Art. 81 Abs. 1 lit. a BGG), zweitens muss der Beschwerdeführer ein rechtlich geschütztes Interesse geltend machen können (Art. 81 Abs. 1 lit. b BGG).

5 Für die vorliegend interessierende Thematik ist das Erfordernis der Verfahrensteilnahme unproblematisch und insofern für die folgende Betrachtungen nicht von weiterem Interesse.

\section{Grundsatz: Kein rechtlich ge- schütztes Interesse}

6 Die Anforderungen an das rechtlich geschützte Interesse des Privatklägers ergeben sich in erster Linie aus Art. 81 Abs. 1 lit. b Ziff. 5 BGG. Demnach ist der Privatkläger zur Beschwerde in Strafsachen legitimiert, «wenn der angefochtene Entscheid sich auf die Beurteilung [der] Zivilansprüche auswirken kann». Zivilforderungen sind Forderungen, die in einem Zusammenhang mit der zu beurteilenden Straftat stehen und ihre Grundlage im 
Zivilrecht haben. $4 \mathrm{Zu}$ denken ist dabei insbesondere an die Verschuldenshaftung nach Art. 41 ff. OR.5 Bei Schädigungen durch den Staat entstehen im Gegensatz zu den durch Privatpersonen verursachten Schäden keine Ersatzforderungen nach Art. $41 \mathrm{ff}$. OR, sondern nach Massgabe des einschlägigen Staatshaftungsgesetzes. Das Bundesgericht hat solche haftungsrechtlichen Ansprüche in ständiger Rechtsprechung als öffentlichrechtliche Forderungen qualifiziert. ${ }^{6}$ Infolgedessen können sich angefochtene Entscheide in Strafverfahren gegen Beamte auf öffentlich-rechtliche, nicht aber auf zivilrechtliche Forderungen auswirken. Mangels Auswirkungen auf die eben gerade inexistenten - Zivilforderungen verwehrt das Bundesgericht den Opfern staatlicher Gewalt die Legitimation zur Beschwerde in Strafsachen, dies gestützt auf den Wortlaut von Art. 81 Abs. 1 lit. b Ziff. 5 BGG. Diese Rechtsprechung ist derart gefestigt, dass das Bundesgericht auf entsprechende Beschwerden teilweise wegen offensichtlicher Unzulässigkeit im Verfahren nach Art. 108 Abs. 1 lit. a BGG nicht eintritt.7

4 BGE 125 IV 163; BGer 1B_6o/2012 vom 13.2.2012, E. 2; Yves Donzallaz, Loi sur le Tribunal fédéral, Bern 2008, 2610; Niklaus Oberholzer, Grundzüge des Strafprozessrechts, 3. Aufl., Bern 2012, 561; so auch Felix Bommer, Offensive Verletztenrechte im Strafprozess, Bern 2006, $51 \mathrm{f}$.

5 BGE 138 IV 87 f.; BGE 125 IV 163; Lorenz Droese, Die Zivilklage nach der schweizerischen Strafprozessordnung, in: Haftpflichtprozess 2011, hrsg. von W. Fellmann/S. Weber, Zürich 2011, 37, 44 f.

6 BGE 131 I 461; BGE 125 IV 164; zuletzt explizit in BGer 6B_945/2013 vom 23.5.2014, E.3.4.

7 So etwa BGer 6B_1107/2014 vom 10.12.2014; BGer 6B_905/2014 vom 18.11.2014; BGer 6B_948/2014 vom 11.11.2014; BGer 6B_228/2014 vom 8.9.2014; BGer 6B_445/2014 vom 2.9.2014; BGer 6B_693/2014 vom 28.8.2014.
7 Die Legitimation für Opfer staatlicher Gewalt könnte sich sodann aus der Generalklausel in Art. 81 Abs. 1 lit. b BGG ergeben. ${ }^{8}$ Allerdings verneint das Bundesgericht in Bezug auf den Privatkläger mit öffentlich-rechtlichen Forderungen ein rechtlich geschütztes Interesse im Sinne der Generalklausel.9 Gemäss Bundesgericht steht der Strafverfolgungsanspruch alleine dem Staat $\mathrm{zu}$, der Privatkläger kann kein solches Interesse geltend machen. ${ }^{10}$ Das in solchen Fällen bloss praktische Interesse an der Verfolgung und Bestrafung genügt nicht. ${ }^{11}$ Wenn das Bundesgericht vom fehlenden Strafverfolgungsinteressen auf die fehlenden rechtlich geschützten Interessen des Privatklägers schliesst, verneint es gleichzeitig implizit andere rechtlich geschützte Interessen. Darauf ist zurückzukommen.

8 Im Grundsatz bedeutet dies: Der Strafkläger i.S.v. Art. 119 Abs. 2 lit. a StPO existiert im bundesgerichtlichen Verfahren nicht, weil das Bundesgericht für die Beschwerdelegitimation des Privatklägers in jedem Fall Auswirkungen auf Zivilforderungen verlangt. ${ }^{12} \mathrm{Ob}$ der Zivilkläger zur Beschwerde legitimiert ist, entscheidet sich sodann nach der Art des Schädigers. Nur wer eine Ersatzforde-

8 Art. 81 Abs. 1 lit. b BGG: «Zur Beschwerde in Strafsachen ist berechtigt, wer [...] ein rechtlich geschütztes Interesse an der Aufhebung oder Änderung des angefochtenen Entscheids hat, insbesondere: $[\ldots] »$.

9 BGer 6B_945/2013 vom 23.5.2014, E. 3.3.2.

10 BGer 6B_945/2013 vom 23.5.2014, E. 3.3.2; ferner BGE 128 I 220; BGE 120 Ia 163; BGer 6B_386/2014 vom 13.5.2014, E. 2.2.

11 BGE 455 I 458; BGer 6B_281/2010 vom 30.4.2010, E. 3; Heinz Aemisegger/Annette Dolge, in: Bundesgerichtsgesetz, Praxiskommentar, hrsg. von Karl Spühler/Heinz Aemisegger/Annette Dolge/Dominik Stock, 2. Aufl., Zürich/St. Gallen 2013, Art. $81 \mathrm{~N} 3$.

12 BGer 6B_1168/2014 vom 13.2.2015, E. 1.2; BGer 6B_481/2014 vom 13.8.2014, E. 5 . 
rung gestützt auf Privatrecht geltend machen kann, kann ans Bundesgericht gelangen. Beim Opfer staatlicher Gewalt ist dies mangels zivilrechtlicher Forderungen nicht der Fall. Mit anderen Worten: Der Umfang des Rechtsschutzes bemisst sich nach dem zugrundeliegenden Haftungsgesetz. Im Ergebnis existieren zwei Klassen von Opfern. ${ }^{13}$

\section{Die ausnahmsweise Zulassung}

9 Trotz teilweise stossender Fälle ${ }^{14}$ und einer bewegten Gesetzgebungsgeschichte ${ }^{15}$ liess sich das Bundesgericht nicht zu einer Kehrtwende bewegen. Die Opferlegitimation in Verfahren gegen Beamte erfuhr nur punktuelle Erweiterungen. Diese wurde auf Grundlage der bereits existierenden Star-Praxis (a) zunächst erweitert (b). Daran anknüpfend sprach das Bundesgericht dem Opfer staatlicher Gewalt ausnahmsweise ein rechtlich geschütztes Interesse in der Sache zu (c).

\section{a) Die Star-Praxis}

10 Die sog. «Star-Praxis» ist Ausgangspunkt der vorliegend interessierenden Entwicklung. Namenspatin für diese Praxis war die Beschwerdeführerin im Entscheid BGE 114 Ia 307: Die STAR Unterhaltungsbetriebe $\mathrm{AG}$, die unter anderem Cabarets betrieb, rekurrierte gegen einen Entscheid des Zürcher Regierungsrates.

13 So auch Etienne Vogel, Ungleiche Verkehrsopfer, in: NZZ vom 26.5.2012, 18, bezüglich den Entscheid BGer 6B_127/2010 vom 15.4.2010.

14 Vgl. etwa BGE 128 IV 188 (Tod bei Ausschaffung); BGE 125 IV 161 (Gelähmte Beine nach Schusswaffengebrauch); BGer 6B_380/2007 vom 13.11.2007 (tödlicher Verkehrsunfall im Baustellenbereich); BGer 6P.84/2004 und 6S.230/2004 vom 11.12.2005 (Beinamputation nach Anhaltung eines Unschuldigen).

15 Ausführlich: Marc Thommen, in: Basler Kommentar Bundesgerichtsgesetz, hrsg. von Marcel Alexander Niggli/Marianne Heer/Hans Wiprächtiger, 2. Aufl., Basel 2011, Art. 81 N 24 f.
Im Bestreben, das Sexgewerbe einzudämmen, senkte der Zürcher Regierungsrat die Bewilligungsquote für das Cabaret «Red Lips» von 5 auf 4 Bewilligungen pro Monat. ${ }^{16}$ Die STAR Unterhaltungsbetriebe AG gelangte daraufhin ans Bundesgericht und machte erfolglos ein durch die Handels- und Gewerbefreiheit geschütztes rechtliches Interesse geltend. Das Bundesgericht trat auf die Beschwerde dennoch ein, indem es - mit einer in der Zwischenzeit etliche Male wiederholten Formulierung - festhielt: «Unbekümmert um die fehlende Legitimation in der Sache selbst kann der Beschwerdeführer eine Verletzung der Verfahrensgarantien geltend machen, deren Missachtung eine formelle Rechtsverweigerung darstellt. Das nach Art. 88 OG erforderliche rechtlich geschützte Interesse ergibt sich diesfalls nicht aus einer Berechtigung in der Sache, sondern aus der Berechtigung, am Verfahren teilzunehmen.»17 Wie das Bundesgericht bereits in früheren Entscheiden ${ }^{18}$ festhielt, ist die Rüge in solchen Fällen auf schwerwiegende Verletzungen von Verfahrensrechten beschränkt, deren Missachtung eine formelle Rechtsverweigerung darstellt. ${ }^{19}$ Eine Legitimation in der Sache gewährt die Star-Praxis nicht, eine inhaltliche Überprüfung des angefochtenen Entscheids ist ausgeschlossen. $\mathrm{Zu}$ diesem Schluss kam das Bundesgericht auch im vorliegenden Fall: Die kantonalen Behörden hatten der Beschwerdeführerin das rechtliche Gehör nicht verweigert, das Bundesgericht wies die Beschwerde entsprechend ab. ${ }^{20}$

16 BGE 114 Ia 308.

17 BGE 114 Ia 312 f.; unter neuem Recht: BGE 133 I $198 \mathrm{f}$.

18 So etwa BGE 94 I 555 und BGE 99 Ia 108 f.

19 BGE 114 Ia 313.

20 BGE 114 Ia 315. 
11 Angesichts der Beschränkung auf schwerwiegende Verletzungen von Verfahrensrechten ist klar: Oftmals ist die Star-Praxis keine Lösung für Opfer staatlicher Gewalt; sie ist aber immerhin wie nachfolgend gezeigt - Ausgangspunkt einer erweiterten Legitimation für Opfer.

\section{b) Die erweiterte Star-Praxis: Der Fall Assenov}

Die Legitimation von Opfern staatlicher Gewalt vor Bundesgericht erfuhr eine erste Erweiterung dank der Rechtsprechung des EGMR. Ausgangspunkt dieser Erweiterung ist der Fall Assenov und Andere v. Bulgarien. ${ }^{21}$ Der EGMR hatte $\mathrm{zu}$ beurteilen, ob die bulgarischen Behörden mit der unterlassenen Untersuchung von erhobenen Missbrauchsvorwürfen das Folterverbot (Art. 3 EMRK) und das Recht auf wirksame Beschwerde (Art. 13 EMRK) verletzt hatten. Der Gerichtshof kam zum Schluss, Art. 3 EMRK vermittle dem Einzelnen nicht nur ein Abwehrrecht gegenüber dem Staat, sondern begründe auch Untersuchungspflichten, die durch den Staat zu gewährleisten seien. ${ }^{22}$ Aufgrund der ungenügenden Untersuchung der Misshandlungsvorwürfe erkannte der EGMR auf eine Verletzung von Art. 3 und Art. 13 EMRK.

Das Bundesgericht übernahm die vom EGMR im Fall Assenov und Andere $v$. Bulgarien entwickelte Rechtsprechung mit dem Entscheid BGE 131 I 455. Beim

21 EGMR vom 28.10.1998, Assenov und Andere $v$. Bulgarien; vgl. Marc Thommen, in: Basler Kommentar Strafprozessordnung, hrsg. von Marcel Alexander Niggli/Marianne Heer/Hans Wiprächtiger, 2. Aufl. Basel 2014, Art. 3 N 26.

22 EGMR vom 28.10.1998, Assenov und Andere $v$. Bulgarien, § 102.
Beschwerdeführer handelte es sich um einen türkischen Staatsangehörigen, der in schwer alkoholisiertem Zustand von Polizisten gewaltsam verhaftet wurde. Die vom Beschwerdeführer verlangte Untersuchung gegen die Polizeibeamten wurde eingestellt. Die Beschwerdeinstanz schützte den Einstellungsentscheid. ${ }^{23}$ Vor Bundesgericht machte der Beschwerdeführer eine Verletzung von Art. 3 und 13 EMRK geltend. Mit Verweis auf die bisherige Rechtsprechung verweigerte das Bundesgericht dem Beschwerdeführer die Legitimation in der Sache. Es anerkannte in Anwendung der StarPraxis indessen ein rechtlich geschütztes Interesse des Beschwerdeführers, soweit dieser Rechte geltend machte, die ihm seiner Prozessstellung wegen zustehen. ${ }^{24}$

14 In seinen Erwägungen verweist das Bundesgericht auf Art. 10 Abs. 3 BV und auf die vom EGMR aus Art. 3 EMRK abgeleiteten prozessualen Garantien. Wer in vertretbarer Weise ${ }^{25}$ vorbringt, er sei von Beamten in erniedrigender oder unmenschlicher Weise behandelt worden, hat einen Anspruch auf eine vertiefte, prompte Untersuchung ${ }^{26}$ der Vorwürfe. ${ }^{27}$ Art. 3 EMRK ist auch dann verletzt, wenn eine in vertretbarer Weise geltend gemachte Verletzung von Art. 3 EMRK gar nicht oder nur in ungenügender Weise

23 BGE 131 I $455 \mathrm{ff}$.

24 BGE 131 I 461.

25 Vgl. EGMR vom 28.10.1998, Assenov und Andere v. Bulgarien, § 102: «an arguable claim»; EGMR vom 24.9.2013, Dembele v. Schweiz, § 62: «soutient de manière défendable».

26 Vgl. EGMR vom 28.10.1998, Assenov und Andere $v$. Bulgarien, § 102: "an effective official investigation»; EGMR vom 24.9.2013, Dembele $v$. Schweiz, § 62: «une enquête officielle effective»; a.a.O., § 63: "L'enquête [...] doit être à la fois rapide et approfondie."

27 BGE 131 I 464. 
untersucht wird. ${ }^{28}$ Verhielte es sich anders, würde Art. 3 EMRK mit seiner fundamentalen Funktion letztlich seines Inhalts entleert. ${ }^{29}$ In Bezug auf die gerügte Verletzung von Art. 13 EMRK kam das Bundesgericht zum Schluss, dass die kantonalen Behörden mit der mangelhaften Untersuchung nicht nur Art. 3 EMRK, sondern auch Art. 13 EMRK verletzten. ${ }^{30}$

sist unklar, wie weit das Bundesgericht mit dem Entscheid BGE 131 I 455 die Legitimation tatsächlich erweitern wollte. Legitimiert war der Beschwerdeführer nur aufgrund der Star-Praxis und nicht in der Sache selbst. ${ }^{31}$ In seinen Erwägungen nahm das Bundesgericht dennoch detailliert Stellung zu den vorgenommenen Untersuchungen und hiess die Beschwerde schliesslich gut - nicht, weil keine Untersuchung stattfand, sondern weil dieselbe mangelhaft war. ${ }^{2}$ Die Legitimation fusst somit auf der Star-Praxis und der Verweigerung von prozessualen Rechten, tatsächlich erstreckt sich die anschliessende Überprüfung des vorinstanzlichen Entscheids aber auf minderschwere Verletzungen von prozessualen Rechten. Fest steht immerhin: Der Entscheid BGE 131 I 455 verbesserte den Rechtsschutz von Opfern staatlicher Ge-

28 BGE 131 I 466; Frank Meyer/Marta Wieckowska, Die Rechtsprechung des EGMR in Strafsachen im Jahr 2011, in: forumpoenale 2012, 116, 123.

29 BGE 131 I 462; vgl. auch EGMR vom 28.10.1998, Assenov und Andere v. Bulgarien, § 102; mit derselben Argumentation bereits EGMR vom 27.9.1995, McCann und Andere $v$. Vereinigtes Königreich, § 161, betreffend Art. 2 EMRK.

3o BGE 131 I 466; zum unklaren Verhältnis von Art. 3 und 13 EMRK: Marten Breuer, in: EMRK, Konvention zum Schutz der Menschenrechte und Grundfreiheiten, Kommentar, hrsg. von Ulrich Karpenstein/Franz C. Mayer, München 2012, Art. 13 N 39.

31 BGE 131 I 461.

32 BGE 131 I $464 \mathrm{ff}$. walt insoweit, als er eine bundesgerichtliche Überprüfung von Nichtanhandnahmen, Einstellungen und Freisprüchen zumindest in schweren Fällen ermöglichte.

\section{c) Legitimation «sur le fond»}

16 Mit dem Entscheid 6B_274/2009 vom 16. Februar 2010 erweiterte das Bundesgericht die in BGE 131 I 455 entwickelte Rechtsprechung. 33

17 In diesem Entscheid gelangte ein Häftling aufgrund eines Vorfalls in der Haftanstalt «Plaine de l'Orbe » ans Bundesgericht: Mehrere Vollzugsbeamte drückten den Beschwerdeführer gewaltsam zu Boden und fesselten ihn mit Handschellen, weil letzterer zuvor unter anderem das Inventar seiner Zelle zerstört hatte. Die vom Häftling geforderte Untersuchung des Vorfalls wurde nicht durchgeführt.34 Der Häftling wehrte sich erfolglos gegen die Nichtanhandnahme und gelangte ein erstes Mal ans Bundesgericht. Im Lichte der Rechtsprechung von BGE 131 I 455 hiess das Bundesgericht die Beschwerde gut und wies die kantonalen Instanzen an, die Untersuchung gegen die beteiligten Vollzugsbeamten aufzunehmen und gegebenenfalls zur Anklage zu bringen. 35 Die daraufhin eröffnete Untersuchung wurde eingestellt, worauf der Häftling erneut ans Bundesgericht gelangte. Das Bundesgericht hielt in seinem neuerlichen Entscheid fest, Art. 13 der

33 BGer 6B_274/2009 vom 16.2.2010; die entsprechende Rechtsprechung fand 4 Jahre später Eingang in die amtliche Sammlung mit dem Entscheid BGE 138 IV 86; zuvor bereits bestätigt in BGer 6B_364/2011 vom 24.10.2011; BGer 6B_688/2010 vom 21.10.2010.

34 BGer 6B_274/2009 vom 16.2.2010, Sachverhalt A.

35 BGer 6B_319/2007 vom 19.9.2007, E. 3. 
UNO-Konvention gegen Folter ${ }^{6}$ vermittle den Opfern staatlicher Gewalt nicht nur ein Recht auf eine angemessene, vertiefte Untersuchung, sondern gegebenenfalls auch einen Anspruch auf angemessene Bestrafung der Täter. ${ }^{37}$ Entsprechend habe der Beschwerdeführer ein rechtlich geschütztes Interesse i.S.v. Art. 81 Abs. 1 lit. b BGG, sodass er in der Sache selbst legitimiert sei. ${ }^{8} 8$ Das Bundesgericht leitete den entsprechenden Anspruch im Vergleich zu BGE 131 I 455 nicht nur aus Art. 10 Abs. 3 BV sowie den Art. 3 und 13 EMRK her, sondern zusätzlich aus Art. 7 IPBPR und der UNOKonvention gegen Folter. Das rechtlich geschützte Interesse ergab sich in diesem Fall nicht aus der Berechtigung, am Verfahren teilzunehmen, sondern unmittelbar aus den angeführten Normen. 39

Mit dem Entscheid 6B_274/2009 war ein Opfer staatlicher Gewalt erstmals in der Sache selbst legitimiert. Diese Recht-

36 Übereinkommen gegen Folter und andere grausame, unmenschliche oder erniedrigende Behandlung oder Strafe, abgeschlossen in New York am 10.12.1984, für die Schweiz in Kraft getreten am 26.6.1987 (SR 0.105).

37 BGer 6B_274/2009 vom 16.2.2010, E. 3.1.2.1; dies gilt sogar dann, wenn das Opfer entschädigt wurde für die geltend gemachte Misshandlung, a.a.O.; zur Bestrafungspflicht vgl. auch EGMR vom 31.5.2011, Derman v. Türkei, § 27; Kollisionen mit dem Opportunitätsprinzip sind in diesen Fällen hinzunehmen, Meyer/Wieckowska (Fn. 28), 124.

38 BGer 6B_274/2009 vom 16.2.2010, E. 3.1.2.1 in fine: «qualité pour recourir sur le fond »; vgl. auch BGer 6B_559/2013 vom 27.1.2014, E. 2.3: «freien bundesgerichtlichen Überprüfung des angefochtenen Urteils»; Heinz Aemisegger, Zur Umsetzung der Schweizerischen Strafprozessordnung, in: Liber amicorum für Andreas Donatsch, Im Einsatz für Wissenschaft, Lehre und Praxis, hrsg. von Angela Cavallo et al., Zürich/Basel/Genf 2012, 251, 261, m.w.H.

39 BGer 6B_274/2009 vom 16.2.2010, E 3.1.2.1; vgl. ferner BGer 1B_272/2011 vom 22.3.2012, E. 2.5; BGer 1B_10/2012 vom 29.3.2012, E. 1.2.4. sprechung wurde inzwischen mehrfach bestätigt.40 Die Erweiterung der Legitimation ist insofern bemerkenswert, als das Bundesgericht in diesem Entscheid für die Legitimation im Wesentlichen dieselben Argumente anführte wie bereits im Entscheid BGE 131 I $455.4^{11}$ Die Gründe für den bundesgerichtlichen Richtungswechsel mögen offen bleiben, klar ist immerhin: Opfer staatlicher Gewalt sind in Fällen glaubhaft vorgebrachter Misshandlungsvorwürfe in der Sache selbst beschwerdelegitimiert. 42

\section{Konsequenzen für das Opfer staat- licher Gewalt}

19 Die ausnahmsweise Legitimation in der Sache von Opfern staatlicher Gewalt ist zu begrüssen. Trotzdem existieren weiterhin Defizite für die Opfer staatlicher Gewalt. Zum einen ist eine Verletzung von Art. 3 EMRK nicht ohne weiteres gegeben (1.). Zum anderen droht eine Schlechterstellung aufgrund der Korrumpierung des Zivilrichters (2.) und der Strafbehörden (3.). Sodann benachteiligt die Notwendigkeit eines separaten Staatshaftungsverfahrens die Opfer staatlicher Gewalt gegenüber den Opfern privater Gewalt (4.). Wie nachfolgend dargelegt, kann man angesichts dieser

40 Vgl. etwa BGer 6B_479/2013 vom 30.1.2014, E. 1. 2; BGer 6B_559/2013 vom 27.1.2014, E. 2.3; BGer 6B_769/2013 vom 16.1.2014, E. 1.3; BGer 6B_364/2011 vom 24.10.2011, E. 2.2; diese Rechtsprechung scheint inzwischen gefestigt, sodass das Bundesgericht die Legitimation in solchen Fällen ohne weiteres bejaht, vgl. BGer 6B_797/2013 vom 27.3.2014, E. 1.

41 Vgl. BGE 131 I 464 und BGer 6B_274/2009 vom 16.2.2010, E. 3.1.2.2 in fine.

42 Dies muss im Übrigen mit analoger Begründung auch bei einer geltend gemachten Verletzung von Art. 2 EMRK gelten, vgl. EGMR vom 27.9.1995, McCann und Andere $v$. Vereinigtes Königreich; EGMR vom 7.2.2006, Scavuzzo-Hager und Andere v. Schweiz; so auch Aemisegger (Fn. 38), 261. 
Defizite nicht ernsthaft von einer OpferGleichbehandlung sprechen.

\section{Grenzen der erniedrigenden Be- handlung}

20 Um als Opfer staatlicher Gewalt in der Sache zur Beschwerde ans Bundesgericht legitimiert zu sein, bedarf es - wie gesehen - der glaubhaften Geltendmachung einer erniedrigenden, unmenschlichen oder folternden Behandlung i.S.v. Art. 3 EMRK.

21 Die erniedrigende Behandlung ist zwar die schwächste Form einer Konventionsverletzung nach Art. 3 EMRK 43 - trotzdem ist eine solche nicht ohne weiteres gegeben. Notwendig ist erstens eine minimale Schwere. Ob diese minimale Schwere erreicht ist, hängt in der Regel von den konkreten Umständen ab, etwa die Dauer der Misshandlung, die physische und psychische Wirkung auf den Betroffenen, das Alter und Geschlecht des Betroffenen sowie dessen allgemeiner Gesundheitszustand.44 Nur ausnahmsweise kommt es auf die konkreten Umstände nicht an, wenn bereits die Situation an sich eine Verletzung von Art. 3 EMRK darstellt.45 Im Zusammenhang mit Polizeigewalt etwa nimmt der EGMR bereits dann eine unmenschliche Behandlung an, wenn der Einsatz von Gewalt das unbedingt notwendige Mass überschreitet. ${ }^{6}$ Eine erniedrigende Behandlung im Sinne von Art. 3 EMRK

43 Stefan Sinner, in: Karpenstein/Mayer (Fn. 30), Art. 3 N 5; BGer 6B_559/2013 vom 27.1.2014, E. 2.4.

44 BGE 134 I 226; Grabenwarter/Pabel (Fn. 43), 163 , m.w.H.

45 EGMR vom 28.7.1999, Selmouni v. Frankreich, § 103.

46 EGMR vom 24.9.2013, Dembele v. Schweiz, §§ 41-42, m.w.H.; BGer 1B_729/2012 vom 28.5.2013, E. 2.1. muss zweitens grundsätzlich vorsätzlich, die Erniedrigung und Demütigung des Opfers beabsichtigt sein. 47

22 Im Ergebnis bleiben Opfer minderschwerer oder fahrlässiger staatlicher Gewalt vom Anwendungsbereich von Art. 3 EMRK und damit von der Legitimation in der Sache ausgeschlossen. $4^{8}$ Der enge Anwendungsbereich von Art. 3 EMRK wiegt umso schwerer, als Übergriffe durch Polizisten und Strafvollzugsbeamte nicht die einzigen Fälle staatlicher Gewalt sind. Die Fälle von minderschwerer oder fahrlässiger staatlicher Gewalt sind breit gefächert: $\mathrm{Zu}$ Opfern werden Personen auch durch das Personal in öffentlichen Spitälern 49, durch Chauffeure von öffentlichen Verkehrsbetrieben ${ }^{50}$ oder durch kantonale BaustellenVerantwortliche51. Im Ergebnis gewährt die bundesgerichtliche Rechtsprechung daher nur einem Bruchteil der Opfer staatlicher Gewalt einen tatsächlich verbesserten Rechtsschutz.

\section{Der gebundene Zivilrichter}

23 In seinen Entscheiden führt das Bundesgericht die inexistenten Zivilforderungen an. Art. 81 Abs. 1 lit. b Ziff. 5 BGG verlangt indessen nicht nur vorhandene $\mathrm{Zi}$ vilforderungen, sondern auch Auswir-

47 BGer 1B_32/2014 vom 24.2.2014, E. 3.1; BGer 6B_274/2009 vom 16.2.2010, E. 3.1.2.2; teilweise reicht bereits das subjektive Empfinden des Opfers zur Qualifikation als erniedrigende oder unmenschliche Behandlung, Sinner, in: Karpenstein/Mayer (Fn. 30), Art. 3 N 8 m.w.H.

48 Ausgeschlossen bleiben auch Geschädigte i.S.v. Art. 115 StPO, weil Art. 3 EMRK nur Opfer i.S.v. Art. 116 StPO schützt.

49 BGer 6B_353/2010 vom 31.5.2010; BGer 6B_357/2010 vom 25.5.2010; BGer 6B_480/2007 vom 31.1.2008.

50 BGer 6B_1059/2013 vom 19.2.2014; BGer 6B_127/2010 vom 15.4.2010.

51 BGer 6B_380/2007 vom 13.11.2007. 
kungen auf dieselben durch den angefochtenen Entscheid. Art. 53 OR statuiert demgegenüber die Unverbindlichkeit strafrechtlicher Erkenntnisse für den Zivilrichter im Bereich der Schuld und der Schadensbemessung. Zwischen Art. 81 BGG und Art. 53 OR existiert damit scheinbar ein Widerspruch. Die Frage der Unverbindlichkeit von strafrechtlichen Erkenntnissen - oder im Umkehrschluss der Bindung des Zivilrichters ist nicht nur in der Lehre umstritten; selbst innerhalb des Bundesgerichts wurden zeitweise gegenteilige Meinungen zur Bindung des Zivilrichters vertreten. $5^{2}$

Zumindest die neuere bundesgerichtliche Rechtsprechung schliesst eine gewisse Korrumpierung des Zivilrichters nicht aus: Das Bundesgericht erklärt, dass für den Zivilrichter derjenige Sachverhalt massgebend sein könne, den die Strafbehörden festgestellt haben.53 Darüber hinaus verlange Art. 81 Abs. 1 lit. b Ziff. 5 BGG nicht eine tatsächliche Auswirkung. Es genüge bereits die blosse Möglichkeit einer Auswirkung. 54 Dies sei bereits dann der Fall, wenn sich der Zivilrichter faktisch an das Strafurteil gebunden fühlt.55 Auswirkungen auf Zivilforderungen bejahte das Bundesgericht in der Vergangenheit etwa, wenn die Vorinstanzen auf Fahrlässigkeit anstelle von Vorsatz erkannt oder eine Tatbestandsqualifikation verneint hatten. 56

52 Bernard Corboz, Le pourvoi en nullité interjeté par le lésé auprès de la Cour de cassation pénale du Tribunal fédéral, in: SJ 1995, 133, 146.

53 BGE 136 IV 39.

54 BGE 120 IV 57; Corboz (Fn. 52), 146; anders noch BGE 107 II $158 \mathrm{f}$.

55 BGE 119 IV 344.

56 BGE 137 IV 222 f.; BGer 6B_365/2011 vom 30.9.2011, E. 1; Donzallaz (Fn. 4), 2607; BSK BGGThommen (Fn. 15), Art. 81 N 33.
25 Für das Opfer staatlicher Gewalt, das nur öffentlich-rechtliche Forderungen geltend machen kann, ist die Frage des korrumpierten Zivilrichters nur auf den ersten Blick belanglos, denn: In manchen Kantonen entscheidet der Zivilrichter über Ansprüche aus Staatshaftung. $57 \mathrm{Zu}$ dem erklären manche kantonalen Haftungsgesetze das Obligationenrecht als ergänzend anwendbar. ${ }^{8}$ Es ist nicht ersichtlich, inwieweit sich die Situation in diesen Fällen anders darstellen sollte: Der Zivilrichter wird sich am ergangenen Strafurteil orientieren, wie er dies auch bei privatrechtlichen Ansprüchen tun würde. 59 Wenn im Falle der öffentlichrechtlichen Ansprüche nicht eine rechtliche Gebundenheit besteht, dann doch immerhin die Möglichkeit einer faktischen Gebundenheit. ${ }^{60,61}$

\section{Die gebundenen Strafbehörden?}

26 Das Risiko einer Korrumpierung besteht nicht alleine beim Zivilrichter, sondern auch bei den Strafbehörden. In der Literatur wird diese Thematik nur am Rande oder gar nicht angesprochen.

57 Vgl. etwa § 7 HG/LU; § 19 lit. a HG/ZH; Art. 17 Abs. 1 LRECA/VD; Art. $13^{\text {bis VG/SG; } § ~} 6 \mathrm{HG} / \mathrm{BS}$.

${ }_{58} \S 3 \mathrm{HG} / \mathrm{LU}$; § $29 \mathrm{HG} / \mathrm{ZH}$; Art. 8 LRECA/VD; Art. $12 \mathrm{VG} / \mathrm{SG} ; \S 2 \mathrm{HG} / \mathrm{BS}$.

59 Das anerkennt auch das Bundesgericht, es erachtet diesen Umstand aber als «unerheblich», BGer 6B_775/2010 vom 8.3.2011, E. 2.3 in fine; vgl. auch Christian Heierli/Anton K. Schnyder, in: Basler Kommentar Obligationenrecht I, hrsg. von Heinrich Honsell/Nedim Peter Vogt/Wolfgang Wiegand, 5. Aufl., Basel 2011, Art. 53 N 4.

60 So auch BSK BGG-Thommen (Fn. 15), Art. $81 \mathrm{~N}$ 41; vgl. im Übrigen BGE 124 IV 190: Im Zweifelsfall ist zumindest bei Privatklägern mit privatrechtlichen Forderungen von Auswirkungen auf Zivilforderungen auszugehen.

61 In BGer 6B_945/2013 vom 23.5.2014, E. 3.3.1, anerkennt das Bundesgericht diese Argumentation explizit, verneint jedoch angesichts des klaren Wortlauts von Art. 81 Abs. 1 lit. b Ziff. 5 BGG die Legitimation von Opfern staatlicher Gewalt. 
Weil Übergriffe von Straf- und Vollzugsbehörden durch die Strafbehörden desselben Kantons untersucht werden, kann die untersuchende Behörde befangen sein: Je nach Organisation und Grösse der involvierten Behörden werden der beschuldigte Beamte und der untersuchende Staatsanwalt bzw. die urteilenden Richter auch künftig zusammenarbeiten müssen. Es ist naheliegend, dass in diesen Fällen eine Verfahrenseinstellung entgegen dem Grundsatz «in dubio pro duriore» oder eine Sachverhaltswürdigung über den Geltungsanspruch von «in dubio pro reo» hinaus dem zukünftigen Arbeitsklima zuträglicher ist als eine allfällige Verurteilung. ${ }^{62}$

Eine zentralisierte Kontrolle durch das Bundesgericht wäre in solchen Fällen wertvoll. Zum einen, weil das Bundesgericht damit allfällige Organisationsmängel der kantonalen Justizbehörden aufdecken könnte und Opfer staatlicher Gewalt Rechtsschutz erhalten würden. Zum andern aber auch, weil das Bundesgericht allfällige Befangenheitsvorwürfe gegen beteiligte Staatsanwälte und Richter glaubhaft entkräften könnte. Dass sich das Bundesgericht in solchen Fällen selbst als unzuständig erklärt, betrifft daher nicht nur die Opfer staatlicher Gewalt, sondern auch die Glaubwürdigkeit von solchen Untersuchungen und von kantonalen Strafbehörden insgesamt.

62 Marc Thommen, Toujours agité - jamais abattu! Die Opfer staatlicher Gewalt vor Bundesgericht, in: «Toujours agité - jamais abattu!», Festschrift für Hans Wiprächtiger, hrsg. von Marc Heer/ Marcel Alexander Niggli/Marc Thommen, Basel 2011, 81, 84; Jörg Künzli et al., Umsetzung der Menschenrechte in der Schweiz, Eine Bestandesaufnahme im Bereich Freiheitsentzug, Polizei und Justiz, Bern 2013, 55 f.

\section{Ein zweites Verfahren}

29 Die bundesgerichtliche Rechtsprechung wirkt sich für Opfer staatlicher Gewalt nicht alleine auf das Beschwerdeverfahren vor Bundesgericht aus, sondern auch auf die Strafverfahren in den Kantonen und vor dem Bundesstrafgericht. Die restriktive Rechtsprechung schliesst Opfer staatlicher Gewalt sowohl von der Beschwerde in Strafsachen als auch von der Adhäsionsklage aus. Das Bundesgericht hält fest: «Öffentlich-rechtliche Ansprüche, auch solche aus Staatshaftungsrecht, können nicht adhäsionsweise im Strafprozess geltend gemacht werden [...].»63 Für seine Haltung führt das Bundesgericht keine Normen an. Die bundesgerichtliche Rechtsprechung liesse sich indessen auf die Strafprozessordnung stützen: Art. 119 Abs. 2 lit. b StPO spricht von privatrechtlichen Adhäsionsansprüchen, Art. 122 ff. StPO beschreiben sodann die Zivilklage.64 Demgegenüber sind Forderungen aus Staatshaftung wie gesehen öffentlich-rechtlicher Natur. Getreu dem Wortlaut der genannten Artikel sind solche Forderungen demnach nicht adhäsionsfähig und daher in einem separaten Staatshaftungsprozess geltend $\mathrm{zu}$ machen.

30 Begründet wird die fehlende Adhäsionsfähigkeit von öffentlich-rechtlichen Forderungen indessen nicht alleine mit dem Wortlaut des Gesetzes; als Begründung wird auch das System der ausschliesslichen Staatshaftung herangezogen, das sowohl der Bund als auch die meisten Kantone kennen: Im System der aus-

63 BGer 6B_830/2014 vom 11.9.2014, E. 2; vgl. auch BGer 6B_228/2014 vom 8.9.2014, E. 2.2: «[...] une telle prétention [de droit public] ne peut être invoquée dans le procès pénal par voie d'adhésion [...].»

64 Vgl. ferner BBl 20061172 ff. 
schliesslichen Staatshaftung steht dem Opfer ein Ersatzanspruch gegen das Gemeinwesen, jedoch kein direkter Anspruch gegenüber dem fehlbaren Beamten zu.65 Strafrechtliche und haftungsrechtliche Verantwortung fallen demnach auseinander: In einem ersten Schritt entscheidet zunächst ein Strafrichter über die strafrechtliche Verantwortung des Staatsangestellten, nicht aber über die haftungsrechtliche Verantwortung. Schadenersatz- und Genugtuungsansprüche kann das Opfer erst in einem Staatshaftungsprozess gegen das verantwortliche Gemeinwesen geltend machen.

31 Die Notwendigkeit eines zweiten Prozesses stellt für das Opfer staatlicher Gewalt eine erhebliche Mehrbelastung dar, sowohl in finanzieller, zeitlicher als auch psychischer Hinsicht. Darauf ist zurückzukommen.

\section{Gerechtfertigte Ungleichbehand- lung?}

Zusammenfassend sieht sich das Opfer staatlicher Gewalt also nicht alleine mit den Hürden von Art. 3 EMRK und den Korrumpierungsrisiken konfrontiert; es hat auch einen zweiten Prozess anzustrengen.

Auf der Suche nach Erklärungen für die Haltung des Bundesgerichts trifft man in der bundesgerichtlichen Rechtsprechung auf verschiedene Begründungen, welche die Ungleichbehandlung von Opfern privater und staatlicher Gewalt zu rechtfer-

65 BGer 6B_228/2014 vom 8.9.2014, E. 2.2; BGer 6B_775/2010 vom 8.3.2011, E. 2.3; Ulrich Häfelin/Georg Müller/Felix Uhlmann, Allgemeines Verwaltungsrecht, 6. Aufl., Zürich/St. Gallen 2010, 2229. tigen versuchen. Die restriktive Rechtsprechung zur Legitimation von Opfern staatlicher Gewalt begründet das Bundesgericht mit dem staatlichen Strafmonopol (1.), der privilegierten Stellung des Opfers staatlicher Gewalt (2.) und dem Auseinanderfallen von Schädigern und Ersatzpflichtigen (3.). Auch die Geschäftslast des Bundesgerichts könnte eine Rolle spielen (4).

\section{Das Strafmonopol des Staates}

34 Ein erstes Argument, mit dem das Bundesgericht die restriktive Rechtsprechung zur Opfer-Legitimation erklärt, ist das staatliche Strafmonopol. Das Bundesgericht hat verschiedentlich betont, dass das Interesse an der Verfolgung und Bestrafung des Täters alleine dem Staat zukomme. ${ }^{66}$ Ein zu berücksichtigendes Interesse von Opfern und Geschädigten bestehe einzig in materiellen Entschädigungsinteressen, die unabhängig von den Bestrafungsinteressen existieren und diese nicht konkurrieren. ${ }^{67}$ An der Verfolgung und Verurteilung des Täters haben Opfer allenfalls ein tatsächliches, mittelbares Interesse, das keinen Anspruch auf Teilhabe am Verfahren vermittle. ${ }^{68}$ Die Lehre teilt diese Auffassung überwiegend. 69

66 BGE 131 I 458 f.; BGE 128 I 219 f.; BGE 120 Ia 163; BGer 6B_445/2014 vom 2.9.2014, E. 2.1; BGer 6B_1103/2013 vom 7.5.2014, E. 1.1.

67 BGer 1B_134/2008 vom 18.8.2008, E. 1.2.

68 BGE 136 IV 39; BGE 128 I 219 f.; BGE 131 I 458; Yves Jeanneret, La partie plaignante et l'action civile, in: ZStrR 2010, 297, 305, m.w.H. auf die Rechtsprechung.

69 Donzallaz (Fn. 4), 2566; Mark Pieth, Schweizerisches Strafprozessrecht, Grundriss für Studium und Praxis, 2. Aufl., Basel 2012, 96; Peter A. Albrecht, Trojanische Pferde - Wege der Zerstörung des öffentlichen Strafrechts, in: Strafrecht, Strafprozessrecht und Menschenrechte, Festschrift für Stefan Trechsel, hrsg. von Andreas Donatsch/Marc Forster/Christian Schwarzenegger, 
Damit blendet das Bundesgericht nicht nur den Wortlaut von Art. 119 Abs. 2 $\mathrm{StPO}^{70}$ aus, es verkennt mindestens teilweise auch die Situation der Opfer staatlicher Gewalt: Indem die Konstituierung als Zivilkläger bei öffentlich-rechtlichen Forderungen de iure unmöglich ist,71 bleibt die Konstituierung als Strafkläger die einzige Möglichkeit, als Betroffener am Strafverfahren teilzunehmen. Die Verfahrensteilnahme als Strafkläger ist sinnvoll, um im Hinblick auf einen anschliessenden Staatshaftungsprozess eine bestmögliche Ausgangslage zu schaffen. ${ }^{72}$ Es ist nicht auszuschliessen, dass beim Opfer Vergeltungsinteressen vorhanden sind, die kein geschütztes Interesse für die Beschwerde ans Bundesgericht darstellen. Solche Interessen lassen sich im Übrigen auch beim Zivilkläger nicht ausschliessen. Beim Zivilkläger schliessen allfällig vorhandene Vergeltungsinteressen die Beschwerdelegitimation allerdings nicht aus. Sie werden vom Bundesgericht im Rahmen von Art. 81 Abs. 1 lit. b BGG nicht berücksichtigt, solange unter mehreren Interessen wenigstens eines vorhanden ist, das rechtlichen Schutz geniesst. Dasselbe muss für den Strafkläger gelten: Sofern der Strafkläger ein schützenswertes Interesse geltend ma-

Zürich 2002, 645, 652 f.; differenziert: Bommer (Fn. 4), 256; krit. Jabornigg (Fn. 3), 320 f.

70 Art. 119 Abs. 2 StPO: «In der Erklärung kann die geschädigte Person [...] a. die Verfolgung und Bestrafung der für die Straftat verantwortlichen Person verlangen (Strafklage) [...].»

${ }^{71}$ Yves Jeanneret/André Kuhn, Précis de procédure pénale, Bern 2013, 7028; Omar Abo Youssef, Die Legitimation des Geschädigten zur Beschwerde in Strafsachen. Zugleich Bemerkungen zu BGE 136 IV 29, BGE 136 IV 41 und BGer, Urteil v. 15.4.2010, 6B_127/2010, in: forumpoenale 2010, $313,317$.

72 Dies insbesondere unter dem Aspekt, dass die strafrechtlichen Erkenntnisse bei der Beurteilung der Staatshaftungsansprüche wie gesehen berücksichtigt werden können. chen kann, darf nicht alleine auf seine allfälligen Vergeltungsinteressen abstellt werden. Es wäre daher angezeigt, die Interessen der Strafkläger, welchen nur ein öffentlich-rechtlicher Anspruch zusteht, differenzierter zu betrachten und ihnen gegebenenfalls die Legitimation zuzusprechen. 73

\section{Das privilegierte Opfer}

36 Im Hinblick auf Geschädigte mit öffentlich-rechtlichen Forderungen hält das Bundesgericht weiter fest, dass sich diese in einer privilegierten Ausgangslage befinden: Im Gegensatz zum "gewöhnlichen» Beschuldigten sei der Staat stets solvent, die Einbringlichkeit von Schadenersatz- und Genugtuungsforderungen entsprechend nicht gefährdet. Vor diesem Hintergrund rechtfertige es sich nicht, dem Opfer staatlicher Gewalt auch noch die Privilegien der Adhäsionsklage nach Art. 122 ff. StPO zukommen zu lassen. 74

37 Diese Begründung überrascht angesichts des Umstands, dass die Adhäsionsklage unter anderem auf opferrechtliche Anliegen zurückgeht.75 Das Bundesgericht erinnert denn auch wiederholt an den ursprünglichen Zweck des Adhäsionspro-

73 Vgl. Felix Bommer, Ausgewählte Fragen der Strafrechtspflege nach Bundesgerichtsgesetz, in: Pierre Tschannen, Neue Bundesrechtspflege, Auswirkungen der Totalrevision auf den kantonalen und eidgenössischen Rechtsschutz, Bern 2007, 153, 181, der das Argument des staatlichen Strafmonopols im Prinzip zulässt, im Ergebnis aber zweifelt, ob sich damit der Ausschluss jeglichen rechtlichen Interessens rechtfertigen lässt; ebenso BSK BGGThommen (Fn. 15), Art. 81 N 53.

74 BGE 128 IV 192; BGer 6S.155/2002 vom 23.5.2002, E. 2.3.

75 Dominic Zehntner, in: Opferhilfegesetz, Bundesgesetz vom 23. März 2007 über die Hilfe an Opfer von Straftaten, Stämpflis Handkommentar, hrsg. von Peter Gomm/Dominic Zehntner, 3. Aufl., Bern 2009, Einleitung N 6; vgl. Droese (Fn. 5), 39. 
zesses: Die möglichst einfache und rasche Durchsetzung eines Zivilanspruchs gegen den Beschuldigten. ${ }^{76}$ Die Unwägbarkeiten des Zivilprozesses wie auch das Insolvenzrisiko des Beschuldigten verlangten nach einer effizienten Beurteilung von Zivilforderungen, die im $\mathrm{Zu}-$ sammenhang mit den zu beurteilenden Straftaten stehen. ${ }^{77}$ In der Literatur werden weitere Gründe angeführt, die für eine adhäsionsweise Geltendmachung von Zivilforderungen sprechen, so etwa der Sachzusammenhang, wonach die Lebenswirklichkeit auch rechtlich als Einheit beurteilt werden soll. ${ }^{78}$ Verwiesen wird weiter auf das verminderte Kostenrisiko der Adhäsionsklage,79 die Verwendung von durch die Staatsanwaltschaft erhobenen Beweisen zum Nachweis der Adhäsionsforderung ${ }^{80}$ sowie Vorteile beim Gerichtsstand. ${ }^{81}$ Auch die mit einem Gerichtsverfahren verbundene (psychische) Belastung wird als Grund für die adhäsionsweise Behandlung von Zivilforderungen genannt. ${ }^{82}$ Indem das Bundesgericht sich an das fehlende Insolvenzrisiko des Gemeinwesens klammert, blendet es die übrigen Gründe aus, die nach herrschender Lehre für das Adhäsionsverfahren sprechen.

76 BGE 128 IV 192; BGE 120 IV 51; BGE 120 Ia 105.

77 BGE 120 Ia 105; BGE 101 Ia 143; vgl. BSK BGGThommen (Fn. 15), Art. $81 \mathrm{~N} 13$.

78 Bommer (Fn. 4), 36, spricht von einer «Lösung in einem Aufwasch».

79 Oberholzer (Fn. 4), 560; Jürg Domenig, Die Adhäsionsklage im Bündner Strafprozess, Zürich $1990,8$.

8 Droese (Fn. 5), 39; Niklaus Schmid, Handbuch des schweizerischen Strafprozessrechts, 2. Aufl., Zürich/St. Gallen 2013, 703.

81 Domenig (Fn. 79), 9; Pieth (Fn. 69), 98.

82 Vgl. Droese (Fn. 5), 39.
38 Soweit über die Staatshaftungsansprüche in einem Verfahren nach Massgabe der Zivilprozessordnung entschieden wird, ${ }^{83}$ ist der Geschädigte den finanziellen Risiken des Zivilprozesses ausgesetzt. Auch in zeitlicher Hinsicht ist die Notwendigkeit eines zweiten Prozesses schwerlich mit den Zielsetzungen des Opferhilfegesetzes vereinbar, Schadenersatz- und Genugtuungsansprüchen möglichst rasch und effizient zu beurteilen. ${ }^{84} \mathrm{Zu}$ erwähnen ist ausserdem die psychische Belastung von Opfern. Deren Ausmass hängt in erster Linie vom individuellen Empfinden des Opfers ab. ${ }^{85}$ Die Frage, ob ein Privater oder ein Beamter das Opfer schädigte, ist dagegen für die psychische Belastung nicht ausschlaggebend. ${ }^{86} \mathrm{Im}$ Hinblick auf die psychische Belastung existieren daher keinerlei Anhaltspunkte für die «Privilegierung» von Opfern staatlicher Gewalt.

39 Abgesehen von der staatlichen Solvenz knüpfen alle Gründe, die für das Adhäsionsverfahren sprechen, am Opfer und nicht am Schädiger an. Das Opfer staatlicher Gewalt erfährt gegenüber dem Adhäsionskläger eine Mehrbelastung zumindest in finanzieller und zeitlicher Hinsicht. In psychischer Hinsicht lassen sich keine Gründe finden, die für eine Minderbelastung von Opfern staatlicher Gewalt sprechen. Insgesamt kann daher von einem privilegierten Opfer staatli-

83 So etwa § 6 Abs. 1 HG/BS; § 7 HG/LU; Art. 18 LRECA/VD; Art. 13 HG/SH; Art. 19 VG/VS.

84 BBl 1990 II 986; so auch Abo Youssef (Fn. 71), 317.

85 Marianne Schwander, Das Opfer im Strafrecht, Aktuelles und potenzielles Opfer zwischen Recht, Psychologie und Politik, Bern/Stuttgart/Wien 2010, 12.

86 So etwa Zehntner (Fn. 75), Art. 1 N 6 und 11, wonach im Opferhilferecht alleine die Auswirkungen auf das Opfer entscheidend sind. 
cher Gewalt keine Rede sein, die Argumentation des Bundesgerichts läuft ins Leere.

\section{Der ausgeschlossene Staat}

40 Ein dritter Erklärungsversuch für die bundesgerichtliche Haltung findet sich in der Literatur: Ein Teil der Lehre stellt sich auf den Standpunkt, dass Adhäsionsforderungen nur gegen Verfahrensbeteiligte geltend gemacht werden können. Opfer staatlicher Gewalt haben indessen aufgrund der ausschliesslichen Staatshaftung keinen direkten Anspruch gegen den Beschuldigten, sondern allein gegen das - im Strafverfahren nicht vertretene - Gemeinwesen.87 Man gewinnt den Eindruck, dass dem Gemeinwesen nicht zugemutet wird, Ersatzleistungen zu zahlen, ohne vorgängig zur Ersatzforderung Stellung nehmen zu können. Umgekehrt wird es dem Opfer staatlicher Gewalt zugemutet, ein zweites Verfahren anzustreben.

Die Argumentation des ausgeschlossenen Gemeinwesens blendet erstens aus, dass nach Massgabe der meisten Staatshaftungsgesetze das ersatzpflichtige Gemeinwesen Regress auf den betroffenen Staatsangestellten nehmen kann. Der Beschuldigte wird nicht schadlos gehalten; das Gemeinwesen kann die geleisteten Entschädigungen vom Beschuldigten zurückfordern, sofern der Beschuldigte vorsätzlich oder grobfahrlässig handelte. ${ }^{88}$ Der Beschuldigte wird daher in den meisten Fällen im eigenen Interesse dafür sorgen, allfällige Vorbringen seitens des Opfers möglichst zu entkräften, da die vom Gemeinwesen geleisteten Ersatzforderungen am Ende auf ihn zurückfallen können.

42 Zweitens gilt es zu bedenken, dass die Einführung der ausschliesslichen Staatshaftung ein rechtspolitischer Entscheid war, der den Geschädigten die Eruierung des fehlbaren Beamten ersparen und damit die Geltendmachung von Ansprüchen erleichtern sollte.89 Dass dieser rechtspolitische Entscheid gleichzeitig den Zugang zum Bundesgericht für Opfer staatlicher Gewalt verwehren sollte, ist vor diesem Hintergrund schwer nachvollziehbar.

43 Anstatt die Opfer staatlicher Gewalt für die Geltendmachung ihrer öffentlichrechtlichen Forderungen in ein Staatshaftungsverfahren zu verweisen, wäre eine Konzeption denkbar, die der Streitverkündung im Zivilprozess (Art. $78 \mathrm{ff}$. ZPO) nachempfunden ist. Das ersatzpflichtige Gemeinwesen könnte dadurch als streitberufene Partei direkt im Strafverfahren Stellung zu den Ersatzforderungen des Geschädigten Stellung nehmen. Gleichzeitig entfiele ein separates Staatshaftungsverfahren. Dieses Konzept würde allerdings nur funktionieren, wenn öffentlich-rechtliche Forderungen adhäsionsfähig wären, was de lege lata nicht der Fall ist.9o

$87 \overline{\text { Donzallaz (Fn. 56), 2615; Oberholzer (Fn. 4), } 561}$ und 1666; zumindest angetönt in BGer 6B_1168/2014 vom 13.2.2015, E. 1.2.

88 Fall der internen Beamtenhaftung, Häfelin/Müller/Uhlmann (Fn. 65), 2309 und 2315; vgl. etwa Art. 7 VG; § 11 HG/LU; § 15 Abs. 1 HG/ZH; Art. 10 Abs. 1 LRECA/VD.

$89 \mathrm{BBl} 1956 \mathrm{I} 1397$.

90 BGer 6B_228/2014 vom 8.9.2014, E. 2.2; BSK StPO-Goran Mazzucchelli/Mario Postizzi (Fn. 21), Art. 119 N 10. 


\section{Die Geschäftslast}

44 Auch wenn die Geschäftslast vom Bundesgericht in der vorliegend interessierenden Thematik bisher nicht als Argument angeführt wurde, argumentierte das Bundesgericht in der Vergangenheit im Zusammenhang mit der Legitimation von Geschädigten und Privatstrafklägern mit der Geschäftslast.91 Weil mit der Zulassung von Opfern staatlicher Gewalt eine Zunahme der Geschäftslast verbunden wäre, rechtfertigt es sich, auf das Argument der Geschäftslast einzugehen: Im Entscheid BGE 136 IV 29 begründet das Bundesgericht die Verweigerung der Legitimation des Geschädigten mit Verweis auf die eigene Geschäftslast. Mit der $\mathrm{Zu}-$ lassung der Geschädigten zur Beschwerde in Strafsachen wären eine «erhebliche Zunahme von Beschwerden» und damit spürbare Auswirkungen auf die Geschäftslast verbunden..$^{2}$

45 Das Argument der Geschäftslast ist rechtsstaatlich bedenklich: Mit der Gewaltenteilung sind die Kompetenzen zwischen Gesetzgeber und Bundesgericht klar geregelt. Das Bundesgericht hat im Rahmen seiner internen Organisation für eine möglichst effiziente Bewältigung der eingehenden Beschwerden zu sorgen. Der Gesetzgeber ist demgegenüber verantwortlich, dem Bundesgericht diejenigen finanziellen und personellen Mittel zur Verfügung zu stellen, die das Bundesgericht für die Bewältigung der Geschäftslast braucht. 93 Es ist daher stos-

92 BGE 136 IV 38.

93 Guisep Nay, Das Bundesgerichtsgesetz im Kontext der Justizreform, in: Menschenrechte, Demokratie und Rechtsstaat, Liber amicorum Luzius Wildhaber, hrsg. von Bernhard Ehrenzeller et al., Zürich/St. Gallen 2007, 1469, 1487. send, dass das Bundesgericht das rechtlich geschützte Interesse in teilweise selektiver Art und Weise zuspricht und damit seine Auslastung zu regulieren versucht.94 Dies umso mehr, als dass Bundesrat und Gesetzgeber explizit auf eine enumerative Aufzählung in Art. 81 Abs. 1 lit. b BGG verzichteten und stattdessen eine Generalklausel mit Regelbeispielen formulierten. 95

46 Abgesehen davon, dass das Argument in der Sache nicht überzeugt, darf es schlicht kein Argument für die Legitimationserteilung oder -verweigerung sein. ${ }^{96}$ Eine Begründung für die fehlende Legitimation von Opfern staatlicher Gewalt ist die Geschäftslast nicht, sodass auch der letzte Erklärungsversuch scheitert.

\section{Fazit}

47 Für die beiden eingangs erwähnten Fussgänger stellt sich die Situation zusammengefasst wie folgt dar: Der Fussgänger, der vom privaten PW angefahren wurde, kann sich als Privatkläger am Verfahren beteiligen, seine Ersatzforderungen adhäsionsweise geltend machen und gegebenenfalls ans Bundesgericht gelangen. Demgegenüber kann sich der Fussgänger, der vom Streifenwagen angefahren wurde, zwar als Strafkläger konstituieren und am kantonalen Verfahren teilnehmen. Beschwerde in Straf-

94 So bereits Marc Thommen/Hans Wiprächtiger, Die Beschwerde in Strafsachen, in: AJP 2006, 651,655 .

95 BBl 2001 4318; vgl. auch die Antwort von BR Evelyne Widmer-Schlumpf auf eine Motion von NR Luzi Stamm während der Behandlung des BGG im Nationalrat, Amtl. Bull NR 2010123.

96 Giusep Nay, Recht haben und Recht bekommen vor Bundesgericht, in: Festschrift für Franz Riklin, hrsg. von Marcel Alexander Niggli/José Hurtado Pozo/Nicolas Queloz, Zürich 2007, 453, 465. 
sachen wird er aller Wahrscheinlichkeit nach aber nicht führen können, der Weg ans Bundesgericht bleibt ihm verwehrt. Der Grund für diese ungleichen Möglichkeiten liegt in der restriktiven Rechtsprechung des Bundesgerichts: Das Bundesgericht qualifiziert Forderungen, die ihre Grundlage in Staatshaftungsgesetzen finden, als öffentlich-rechtliche Ansprüche. Mangels Auswirkungen auf die inexistenten Zivilforderungen sind Opfer staatlicher Gewalt grundsätzlich in der Sache nicht legitimiert. Nur ausnahmsweise sind Opfer staatlicher Gewalt in schweren Fällen über die «Plaine de l'Orbe»-Rechtsprechung zur Beschwerde in Strafsachen legitimiert. Einer Abkehr von dieser restriktiven Rechtsprechung und einer Ausweitung der Beschwerdelegitimation hat sich das Bundesgericht bisher verschlossen.

48 Die Gründe, mit denen das Bundesgericht die verweigerte Legitimation für Opfer staatlicher Gewalt begründet, überzeugen nicht. Weder das unbestrittene Gewaltmonopol des Staates, noch der Umstand, dass das haftpflichtige Gemeinwesen im Strafverfahren nicht Stellung $\mathrm{zu}$ allfälligen Schadenersatzund Genugtuungsforderungen nehmen kann, können die bundesgerichtliche Rechtsprechung ernstlich rechtfertigen. Auch das Argument, wonach Opfer staatlicher Gewalt gegenüber anderen Opfern und Geschädigten privilegiert seien, hält einer näheren Betrachtung nicht stand. Es bleibt das Argument der Geschäftslast, das aber keines sein darf.

Das Opfer ist nicht immer im Recht und so geht es vorliegend denn auch nicht darum, dem Opfer stets Recht zu geben. Die Forderung ist einzig, dass das Bun- desgericht sich nicht in wortklauberischer Weise hinter Legitimationsvoraussetzungen verschanzt. Das Bundesgericht soll angesichts des rechtlich geschützten Interessens von Opfern staatlicher Gewalt auf entsprechende Beschwerden eintreten und einen Entscheid in der Sache fällen. ${ }^{97}$ Es steht ausser Frage, dass mit dem Einbezug von weiteren Personen das weitgehend austarierte System des Strafprozesses zumindest in Teilbereichen eine Neuorientierung erfährt. Wenn aber die grundlegenden Entscheide rechtspolitisch bereits gefällt, im Strafprozess umgesetzt und weitgehend auch in Lehre und Rechtsprechung akzeptiert sind, geht es mit der Gleichstellung von Opfern staatlicher Gewalt im bundesgerichtlichen Verfahren nicht mehr um eine Verlagerung des prozessualen Fokus. Mit der Zulassung von Opfern staatlicher Gewalt zur Beschwerde in Strafsachen werden dem Strafverfahren keine neuen Aufgaben aufgebürdet, sondern ein gesetzgeberischer Entscheid konsequent umgesetzt. Dass sich das Bundesgericht diesem gesetzgeberischen Entscheid bisher widersetzt, ist bedenklich. 98

50 Sowohl die Rechtsprechung als auch der Bundesgesetzgeber haben in den letzten 20 Jahren die Grundlagen geschaffen, um Opfern die Geltendmachung ihrer Interessen zu ermöglichen. Diese Entwicklung sollte nicht vor den Opfern staatlicher Gewalt Halt machen. Es ist daher zu hoffen, dass das Bundesgericht seine restriktive Haltung überdenkt und in Sachen Rechtsschutz alle Opfer gleich behandelt.

97 Vgl. Abo Youssef (Fn. 71), 317.

98 Nay (Fn. 96), 464, spricht von einem Widersetzen gegenüber dem gesetzgeberischen Willen in «unzulässiger Weise». 